\title{
MODELING HEAT DISTRIBUTION WITH THE USE OF A NON-INTEGER ORDER, STATE SPACE MODEL
}

\author{
KRZYSZTOF OPRZĘDKIEWICZ $^{a, *}$, EDYTA GAWIN $^{b}$, WOJCIECH MITKOWSKI $^{a}$ \\ ${ }^{a}$ Department of Automatics and Biomedical Engineering \\ AGH University of Science and Technology, al. A. Mickiewicza 30, 30-079 Kraków, Poland \\ e-mail: \{wojciech.mitkowski,kop\}@agh.edu.pl \\ ${ }^{b}$ Department of Computer Sciences \\ High Vocational School in Tarnów, al. A Mickiewicza 8, 33-100 Tarnów, Poland \\ e-mail: e_gawin@pwsztar.edu.pl
}

\begin{abstract}
A new, state space, non-integer order model for the heat transfer process is presented. The proposed model is based on a Feller semigroup one, the derivative with respect to time is expressed by the non-integer order Caputo operator, and the derivative with respect to length is described by the non-integer order Riesz operator. Elementary properties of the state operator are proven and a formula for the step response of the system is also given. The proposed model is applied to the modeling of temperature distribution in a one dimensional plant. Results of experiments show that the proposed model is more accurate than the analogical integer order model in the sense of the MSE cost function.
\end{abstract}

Keywords: non-integer order systems, heat transfer equation, infinite dimensional systems, Feller semigroups.

\section{Introduction}

Mathematical models of distributed parameter systems obtained on the basis of partial differential equations can be described in an infinite dimensional state space, usually in a Hilbert space, but a Sobolev space can also be applied. This problem has been analyzed by many authors. Fundamentals were given, for example, by Pazy (1983) or Mitkowski (1991), and an analysis of a hyperbolic system in Hilbert spaces was presented by Bartecki (2013). This paper presents also a comprehensive overview of the literature.

The modeling of processes and phenomena hard to describe with the use of other tools is one of the main areas of application of non-integer order calculus. Non-integer models for many physical phenomena were presented by many authors, for example, by Podlubny (1999), Dzielinski et al. (2010), Caponetto et al. (2010), Das (2010), Obraczka (2014), Sierociuk et al. (2015), or Gal and Warma (2016). Analysis of the anomalous diffusion problem with the use of a fractional order approach and semigroup theory was presented, for example, by

* Corresponding author
Popescu (2010). An observability problem for fractional order systems was presented, among others, by N'Doye et al. (2013) or Kaczorek (2016), and controllability was investigated, for instance, by Balachandran (2012; 2014).

It is well known that heat transfer processes can also be modeled with the use of a non-integer order approach. This problem was investigated, for example, by Baeumer et al. (2005), Kochubei (2011), Almeida and Torres (2011), Mitkowski (2011), Obraczka (2014) or Dlugosz and Skruch (2015).

It is important to note that all known models have the form of a transfer function or a partial differential equation. A non-integer order, state space model for the heat transfer process has not been presented yet. This paper gives a proposal of a new, state-space model for the heat transfer process in a one dimensional plant. The idea of this model is based directly on a semigroup model for one-dimensional heat transfer problem.

The paper is organized as follows. First, elementary ideas and definitions are given. Next, the investigated, experimental, infinite order plant and its integer order, semigroup model are presented. Furthermore, the proposal of a non-integer order model and its elementary 
properties are discussed. Finally, the proposed model is verified with the use of experimental results.

\section{Preliminaries}

We will start the set of elementary ideas with recalling the idea of the gamma Euler function (see, e.g., Kaczorek and Rogowski, 2014).

Definition 1. The gamma function is given by

$$
\Gamma(x)=\int_{0}^{\infty} t^{x-1} e^{-t} \mathrm{~d} t .
$$

The idea of the Mittag-Leffler function should be given next. It is a non-integer order generalization of exponential function $e^{\lambda t}$ and plays a crucial role in solution of the fractional order (FO) state equation. The Mittag-Leffler functions are defined below.

Definition 2. The one parameter Mittag-Leffler function is given by

$$
E_{\alpha}(x)=\sum_{k=0}^{\infty} \frac{x^{k}}{\Gamma(k \alpha+1)} .
$$

Definition 3. The two parameter Mittag-Leffler function is given by

$$
E_{\alpha, \beta}(x)=\sum_{k=0}^{\infty} \frac{x^{k}}{\Gamma(k \alpha+\beta)} .
$$

For $\beta=1$, the two parameter function (3) turns into the one parameter function (2).

The fractional-order, integro-differential operator can be described by different definitions, given by Grünvald and Letnikov (GL), Riemann and Liouville $(\mathrm{RL})$, and Caputo (C). All these definitions are given below. With respect to particular additional assumptions, these definitions are equivalent.

Definition 4. The Grünvald-Letnikov definition of the FO operator (Caponetto et al., 2010; Ostalczyk, 2012) is as follows:

$$
{ }_{0}^{G L} D_{t}^{\alpha} f(t)=\lim _{h \rightarrow 0} h^{-\alpha} \sum_{j=0}^{\left[\frac{t}{h}\right]}(-1)^{j}\left(\begin{array}{c}
\alpha \\
j
\end{array}\right) f(t-j h) .
$$

In (4), $\left(\begin{array}{c}\alpha \\ j\end{array}\right)$ is a generalization of the Newton symbol into real numbers:

$$
\left(\begin{array}{l}
\alpha \\
j
\end{array}\right)= \begin{cases}1, & j=0, \\
\frac{\alpha(\alpha-1) \ldots(\alpha-j+1)}{j !}, & j>0,\end{cases}
$$

where $\alpha \in \mathbb{R}$ and $j \in \mathbb{Z}, j \geq 0$.
Definition 5. The Riemann-Liouville definition of the FO operator is given by

$$
{ }_{0}^{R L} D_{t}^{\alpha} f(t)=\frac{1}{\Gamma(N-\alpha)} \frac{\mathrm{d}^{N}}{\mathrm{~d} t^{N}} \int_{0}^{\infty}(t-\tau)^{N-\alpha-1} f(\tau) \mathrm{d} \tau,
$$

where

$$
N-1<\alpha<N
$$

denotes the non-integer order of this operation.

Definition 6. (Kaczorek, 2016) The Caputo definition of the FO operator is as follows:

$$
{ }_{0}^{C} D_{t}^{\alpha} f(t)=\frac{1}{\Gamma(N-\alpha)} \int_{0}^{\infty} \frac{f^{(N)}(\tau)}{(t-\tau)^{\alpha+1-N}} \mathrm{~d} \tau .
$$

where $N-1<\alpha<N$ denotes the non-integer order of this operation.

The non-integer order spatial derivative was given by Riesz and has the following form (see, e.g., Yang et al., 2010).

Definition 7. The Riesz definition of the FO spatial derivative is given by

$$
\frac{\partial^{\gamma} \Theta(x, t)}{\partial x^{\gamma}}=-d_{\gamma}\left({ }_{0} D_{x}^{\gamma}+{ }_{x} D_{1}^{\gamma}\right) \Theta(x, t),
$$

where

$$
d_{\gamma}=\frac{1}{2 \cos \left(\frac{\pi \gamma}{2}\right)}
$$

In (8), ${ }_{0} D_{x}^{\gamma}$ and $x D_{1}^{\gamma}$ denote left- and right-hand Riemann-Liouville derivatives, defined as

$$
\begin{aligned}
& { }_{0} D_{x}^{\gamma}=\frac{1}{\Gamma(2-\alpha)} \frac{\partial}{\partial x} \int_{0}^{x} \frac{\Theta(\xi, t)}{(x-\xi)^{\gamma-1}} \mathrm{~d} \xi \\
& { }_{x} D_{1}^{\gamma}=\frac{1}{\Gamma(2-\alpha)} \frac{\partial}{\partial x} \int_{x}^{1} \frac{\Theta(\xi, t)}{(\xi-x)^{\gamma-1}} \mathrm{~d} \xi .
\end{aligned}
$$

For the RL or C operators, the Laplace transform can be defined as follows (see, e.g., Kaczorek, 2011).

Definition 8. The Laplace transform for the RiemannLiouville operator is given by

$$
\begin{aligned}
\mathcal{L}\left({ }_{0}^{R L} D_{t}^{\alpha} f(t)\right)= & s^{\alpha} F(s), \quad \alpha<0, \\
\mathcal{L}\left({ }_{0}^{R L} D_{t}^{\alpha} f(t)\right)= & s^{\alpha} F(s)-\sum_{k=0}^{n-1} s^{k}{ }_{0} D_{t}^{\alpha-k-1} f(0), \\
& \alpha>0, \quad n-1<\alpha \leq n \in \mathbb{N} .
\end{aligned}
$$


Definition 9. The Laplace transform for the Caputo operator is given by

$$
\begin{aligned}
\mathcal{L}\left({ }_{0}^{C} D_{t}^{\alpha} f(t)\right)= & s^{\alpha} F(s), \quad \alpha<0, \\
\mathcal{L}\left({ }_{0}^{C} D_{t}^{\alpha} f(t)\right)= & s^{\alpha} F(s)-\sum_{k=0}^{n-1} s^{\alpha-k-1}{ }_{0} D_{t}^{k} f(0), \\
& \alpha>0, \quad n-1<\alpha \leq n \in \mathbb{N} .
\end{aligned}
$$

Consequently, the inverse Laplace transform for a non-integer order function is expressed as follows (see, e.g., Kaczorek and Rogowski, 2014):

$$
\begin{array}{r}
\mathcal{L}^{-1}\left[s^{\alpha} F(s)\right]={ }_{0} D_{t}^{\alpha} f(t)+\sum_{k=0}^{n-1} \frac{t^{k-1}}{\Gamma(k-\alpha+1)} f^{(k)}\left(0^{+}\right), \\
n-1<\alpha<n, \quad n \in \mathbb{Z} .
\end{array}
$$

\section{Plant considered and its integer order model}

A simplified scheme of the heat plant considered is shown in Fig. 1. It has the form of a thin copper rod heated with an electric heater of length $\Delta x_{u}$ localized at one end of the rod. An output temperature is measured with the use of four RTD sensors, $\Delta x$ long, located at points $0.3,0.5,0.7$ and 0.9 of the rod length. The construction of the whole experimental system is presented in detail in Section 5. A fundamental mathematical model describing heat

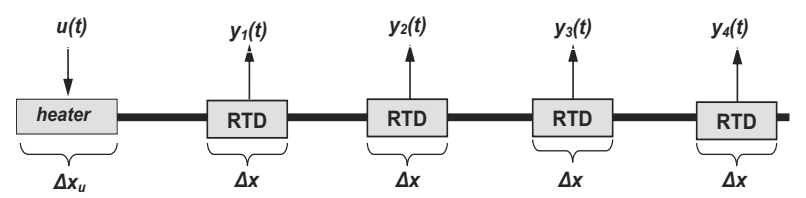

Fig. 1. Simplified scheme of the experimental system.

conduction in the plant is a partial differential equation of the parabolic type with homogeneous Neumann boundary conditions at the ends, the homogeneous initial condition, heat exchange along the length of the rod, and distributed control and observation. This equation was considered, for example, by Oprzedkiewicz $(2003 ; 2005)$ :

$$
\left\{\begin{array}{l}
\frac{\partial Q(x, t)}{\partial t}=a \frac{\partial^{2} Q(x, t)}{\partial x^{2}}-R_{a} Q(x, t)+b(x) u(t) \\
\frac{\partial Q(0, t)}{\partial x}=0, \quad t \geq 0 \\
\frac{\partial Q(1, t)}{\partial x}=0, \quad t \geq 0 \\
Q(x, 0)=0, \quad 0 \leq x \leq 1, \\
y(t)=y_{0} \int_{0}^{1} Q(x, t) c(x) \mathrm{d} x
\end{array}\right.
$$

where $Q(x, t)$ denotes the temperature at time instant $t$ and point $x, R_{a}$ and $a$ denote respectively the coefficients of heat conduction and heat exchange, $b(x)$ denotes the heater function (Curtain and Zwart, 1995), $u(t)$ is an input signal, $c(x)$ is an observation function and $y_{0}$ denotes the steady-state gain of the system.

The heat equation (15) can be shown as an equivalent abstract initial problem in Hilbert space $X=L^{2}(0,1)$ with the standard scalar product. This issue was discussed, for example, by Mitkowski (1991). In our case the abstract form of the heat equation (15) is

$$
\left\{\begin{array}{l}
\dot{Q}(t)=A Q(t)+B u(t), \\
Q(0)=0, \\
y(t)=y_{0} C Q(t),
\end{array}\right.
$$

where

$$
\left\{\begin{array}{l}
A Q=a Q^{\prime \prime}-R_{a} Q, \\
D(A)=\left\{Q \in H^{2}(0,1): Q^{\prime}(0)=0, Q^{\prime}(1)=0\right\}, \\
a, R_{a}>0, \\
H^{2}(0,1)=\left\{w \in L^{2}(0,1): w^{\prime}, w^{\prime \prime} \in L^{2}(0,1)\right\}, \\
C Q(t)=\langle c, Q(t)\rangle, B u(t)=b u(t), \\
\langle w, v\rangle=\int_{0}^{1} w(x) v(x) \mathrm{d} x .
\end{array}\right.
$$

The following set of eigenvectors for the state operator $A$ forms an orthonormal basis of the state space:

$$
h_{i}(x)= \begin{cases}1, & i=0, \\ \sqrt{2} \cos (i \pi x), & i=1,2, \ldots\end{cases}
$$

The discrete spectrum of the state operator for the integer order model $A$ is a set of single, real eigenvalues, which are expressed as follows:

$$
\lambda_{i}=-a \pi^{2} i^{2}-R_{a}, \quad i=0,1,2, \ldots
$$

In the state space basis defined by the set of eigenvectors (18), the operators $A, B$ and $C$ have the following matrix representation:

$$
\begin{gathered}
A=\operatorname{diag}\left\{\lambda_{0}, \lambda_{1}, \lambda_{2}, \ldots\right\}, \\
B=\left[b_{0}, b_{1}, b_{2}, \ldots\right]^{T},
\end{gathered}
$$

where $b_{i}=\left\langle b, h_{i}\right\rangle, b(x)$ denotes the heater function:

$$
b(x)= \begin{cases}1, & x \in\left[0, x_{0}\right], \\ 0, & x \notin\left[0, x_{0}\right] .\end{cases}
$$

The output operator is written as

$$
C=\left[\begin{array}{l}
C_{s 1} \\
C_{s 2} \\
C_{s 3} \\
C_{s 4}
\end{array}\right]
$$


The rows of the output operator $C$ are expressed as follows:

$$
C_{s j}=\left[c_{s j, 0}, c_{s j, 1}, c_{s j, 2}, \ldots\right], \quad j=1,2,3,4,
$$

where $c_{s j, i}=\left\langle c, h_{i}\right\rangle, c(x)$ denotes the output sensor function

$$
c(x)= \begin{cases}1, & x \in\left[x_{1}, x_{2}\right], \\ 0, & x \notin\left[x_{1}, x_{2}\right] .\end{cases}
$$

Coordinates $x_{1}$ and $x_{2}$ depend on the sensor location on the rod and they are equal to

$$
\begin{cases}x=0.3: & x_{1}=\frac{15}{52}, x_{2}=\frac{17}{52}, \\ x=0.5: & x_{1}=\frac{25}{52}, x_{2}=\frac{27}{52}, \\ x=0.7: & x_{1}=\frac{36}{52}, x_{2}=\frac{38}{52} \\ x=0.9: & x_{1}=\frac{46}{52}, x_{2}=\frac{48}{52} .\end{cases}
$$

From (22) and 25), it turns out that the heater function $b(x)$ and the sensor function $c(x)$ are interval constant functions. Assume that the control function $u(t)=\mathbf{1}(t)$. Then the solution to 16 has the form similar to solution for a single output plant presented, for example, by Oprzedkiewicz (2003):

$$
\begin{array}{r}
y_{\mathrm{IO}_{j}}(t)=y_{0_{j}} \sum_{i=1}^{\infty}\left(\frac{e^{\lambda_{i} t}-1}{\lambda_{i}}\right)\left\langle b, h_{i}\right\rangle\left\langle c, h_{i}\right\rangle, \\
j=1,2,3,4 .
\end{array}
$$

The basic features of the discussed parabolic IO system have been analyzed. It can be proved that the state operator $A$ for the considered system is negative, self-adjoint and has a compact inverse operator. For known coefficients $a$ and $R_{a}$, Eqns. (16)-25) provide a good description of the real experimental heat object considered. By "truncating" further elements of infinite-dimensional operators $A, B$ and $C$, we obtain its finite-dimensional approximation, which is a useful tool for numerical modeling of the discussed plant. In this case, the operators $A, B$ and $C$ can be interpreted as matrices. If these parameters are not exactly known, then an interval model can be applied. This problem was presented by Oprzedkiewicz (2003; 2004; 2005).

It is important to note that the approach using semigroup theory presented above can be extended to non-integer order systems. This problem was discussed, for example, by Popescu (2010) or Szekeres and Izsak (2014), and this approach will be applied also to the non-integer order case proposed in the next section.

\section{Non-integer order model of the system}

The proposed non-integer order model with respect to both time and space coordinates is obtained by replacing the first order time difference and the second order spatial difference by suitable non-integer order differences. This is motivated by the fact that the dynamics of the spatial heat distribution along the heater and the rod and along the rod and the sensor are not exactly described by Eqn. (15). The non-integer order differentiation is expected to better describe these processes. Similar issues were studied by Baeumer et al. (2005), Kochubei (2011) or Almeida and Torres (2011).

Assume that the non-integer order difference with respect to time is described by the Caputo definition (7) and non-integer order difference with respect to length is described by the Riesz definition (8). Then the heat transfer equation turns to the following form:

$$
\left\{\begin{array}{l}
{ }^{C} D_{t}^{\alpha} Q(x, t)=a \frac{\partial^{\beta} Q(x, t)}{\partial x^{\beta}}-R_{a} Q(x, t)+b(x) u(t) \\
\frac{\partial Q(0, t)}{\partial x}=0, \quad t \geq 0 \\
\frac{\partial Q(1, t)}{\partial x}=0, \quad t \geq 0 \\
Q(x, 0)=0, \quad 0 \leq x \leq 1 \\
y(t)=y_{0} \int_{0}^{1} Q(x, t) c(x) \mathrm{d} x
\end{array}\right.
$$

where $\alpha, \beta>0$ denote non-integer orders of the system and the other parameters are the same as in the IO model (16). An abstract interpretation of the system (27) was presented by Popescu (2010). Now Eqn. (27) needs to be expressed as a state equation in the Hilbert space analogically to 16 . It has the following form:

$$
\left\{\begin{array}{l}
{ }^{C} D_{t}^{\alpha} Q(t)=A Q(t)+B u(t), \\
Q(0)=0 \\
y(t)=y_{0} C Q(t)
\end{array}\right.
$$

where

$$
\left\{\begin{array}{l}
A Q=a \frac{\partial^{\beta} Q(x)}{\partial x^{\beta}}-R_{a} Q, \\
D(A)=\left\{Q \in H^{\beta}(0,1): Q^{\prime}(0)=0, Q^{\prime}(1)=0\right\}, \\
a, R_{a}>0, \\
C Q(t)=\langle c, Q(t)\rangle, B u(t)=b u(t) .
\end{array}\right.
$$

The eigenvalues of the state operator are expressed as (see Yang et al., 2010)

$$
\lambda_{\beta_{i}}=-a \pi^{\beta} i^{\beta}-R_{a}, \quad i=0,1,2, \ldots
$$

The defined operator $A$ has a discrete spectrum consisting of single eigenvalues $\lambda_{\beta_{i}}$, which are associated with the orthonormal eigenvectors (18) forming a basis in $L^{2}(0,1)$.

Next, a spectrum decomposition of the system 29. can be considered. It is presented below:

$$
{ }^{C} D_{t}^{\alpha} Q(t)=A Q(t)+B u(t)
$$




$$
\begin{gathered}
A h_{i}=\lambda_{i} h_{i}, \\
\left\langle h_{i}, h_{k}\right\rangle= \begin{cases}1, & i=k, \\
0, & i \neq k,\end{cases} \\
{ }^{C} D_{t}^{\alpha} Q=a \frac{\partial^{\beta} Q}{\partial x^{\beta}}-R_{a} Q+B u, \\
{ }^{C} D_{t}^{\alpha} \sum_{i=0}^{\infty} c_{i} h_{i}=a \frac{\partial^{\beta} \sum_{i=0}^{\infty} c_{i} h_{i}}{\partial x^{\beta}}-R_{a} \sum_{i=0}^{\infty} c_{i} h_{i}+\frac{B u}{h_{i}}, \\
{ }^{C} D_{t}^{\alpha} c_{i}=a \frac{\partial^{\beta} c_{i}}{\partial x^{\beta}}-R_{a} c_{i}+b_{i} u .
\end{gathered}
$$

The form of Eqn. (31) implies the decomposition of the system (29) into subsystems related to the different eigenvalues $\lambda_{i}, i=0,1,2,3, \ldots$.

Next, Feller semigroups can be applied (Pazy, 1983; Evans and Jacob, 2007; Popescu, 2010). The semigroup is defined as follows.

Definition 10. A semigroup is a set $S$ coupled with a binary operation $T(T: S \times S \rightarrow S)$ which is associative. That is, $\forall x, y, z \in S, \quad T(T(x, y), z)=T(x, T(y, z))$.

The uniqueness of the solution reveals the semigroup property, which is given by

$$
T(t+s)=T(t) T(s), \quad t, s>0 .
$$

The semigroup property (32) of the family of functions, $\{T(t): t \geq 0\}$, is a composition. Notice that $T(0)$ is the identity operator $\left(I_{d}\right)$. A strongly continuous positive contraction semigroup on $C_{\infty}(S)$ is called a Feller semigroup on $S$. We have a differential representation of the operators which have the form of a Feller semigroup. From the results presented by Popescu (2010), we can conclude that the non-integer order system

$$
{ }^{C} D_{t}^{\alpha} Q(t)=A Q(t)+B u(t), \quad Q(0)=0,
$$

where $0<\alpha<1, t \geq 0$ and $A$ is the generator of the bounded continuous Feller semigroup $T(t)_{t \geq 0}$ in the Hilbert space $H^{\beta}(0,1)$.

Elementary properties of the parabolic IO system have been analyzed and it can be proved that the state operator $A$ for an integer order system described by (20) is negative, self-adjoint and has the compact inverse operator.

The corresponding theorem for $\beta=2$ is also true. A similar theorem, for $\beta \in \mathbb{R}$, is true under certain initial conditions. This has been considered, e.g., by Gal and Warma (2016).

The solution of the state equation (28) can be calculated with the use of the Laplace transform for the $\mathrm{C}$ operator defined by (13) on the assumption that the initial condition is equal to zero: $Q(x, 0)=0,0 \leq x \leq 1$ and the state and control operators are described by (20)-(22).
If we assume that the control signal has the form of the Heaviside function $u(t)=\mathbf{1}(t)$ and apply (14), then we obtain the solution of the state equation (28) in the form

$$
\begin{array}{r}
y_{N I O_{j}}(t)=y_{0_{j}} \sum_{i=1}^{\infty} \frac{\left(E_{\alpha}\left(\lambda_{\beta_{i}} t^{\alpha}\right)-1(t)\right)}{\lambda_{\beta_{i}}}\left\langle b, h_{i}\right\rangle\left\langle c, h_{i}\right\rangle, \\
j=1,2,3,4 .
\end{array}
$$

Notice that the proposed non-integer order model described by (27)- (33) for orders $\alpha=1$ and $\beta=$ 2 turns into the integer order model (15)-(26). Next, the non-integer order model described by (28)-(33) is an infinite dimensional model. Its practical application requires from us the use of its finite dimensional approximation. This can be obtained by dropping further nodes in state equation (28) and consequently calculating the solution (33) as a finite sum expressed by (34). Consequently, operators $A, B$ and $C$ are interpreted as matrices,

$$
\begin{array}{r}
y_{N I O F_{j}}(t)=y_{0_{j}} \sum_{i=1}^{N} \frac{\left(E_{\alpha}\left(\lambda_{\beta_{i}} t^{\alpha}\right)-1(t)\right)}{\lambda_{\beta_{i}}}\left\langle b, h_{i}\right\rangle\left\langle c, h_{i}\right\rangle, \\
j=1,2,3,4 .
\end{array}
$$

In (34), $N$ denotes the dimension of the finite dimensional approximation. In a real situation, it can be estimated with the use of simulations. This will be discussed in the next section.

\section{Experimental results}

Experiments were done with the use of an experimental heat plant shown in Fig. 11 A detailed construction of the whole system is shown in Fig. 2 The length of the rod is equal to $260[\mathrm{~mm}]$. The control signal in the system is the standard current signal $0-20[\mathrm{~mA}]$ given from the analog output of a PLC. This signal is amplified to the range of $0-1.5[\mathrm{~A}]$, and it is the input signal for the heater. The temperature distribution along the length of the rod is measured with the use of RTD sensors. Signals from the sensors are transformed to the standard voltage signal $0-10[\mathrm{~V}]$ with the use of transducers. These signals are next read by analog inputs of the PLC. Data from the PLC are read and stored with the use of SCADA. The whole system is connected via the PROFINET industrial network. The temperature distribution with respect to time and length is shown in Fig. 3.

Experiments were performed with the use of the experimental system shown in Fig. 2. The step response of the model was tested in the time range from 0 to $T_{f}=300[\mathrm{~s}]$ with sampling time $1[\mathrm{~s}]$, and parameters were calculated with the use of the least squares method 


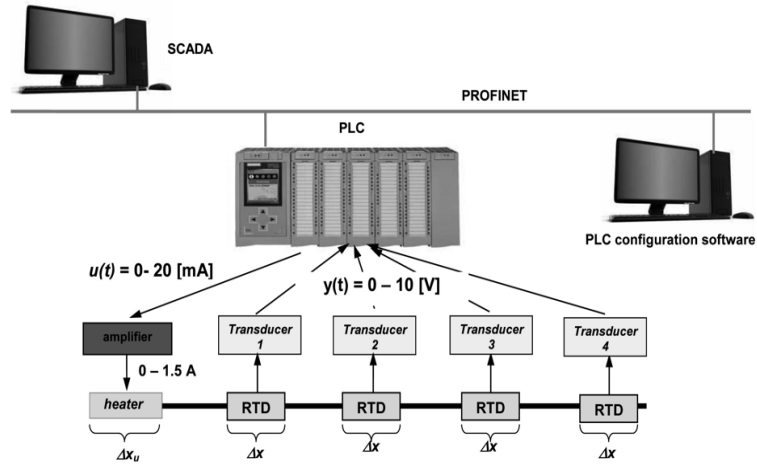

Fig. 2. Structure of the experimental system.

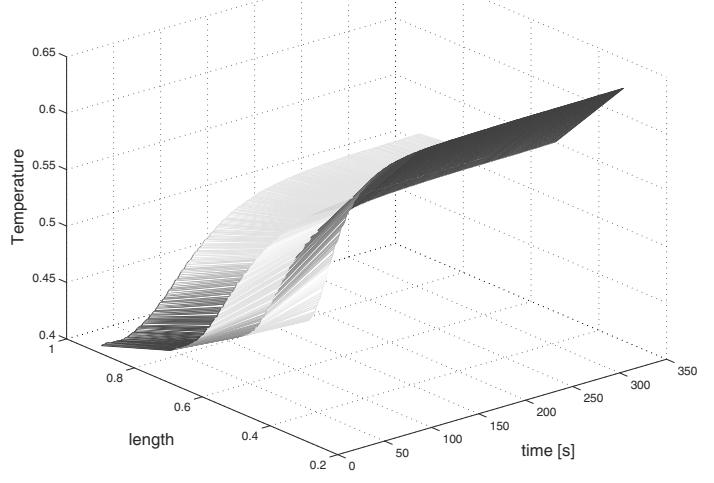

Fig. 3. Spatiotemporal temperature distribution in the plant.

to optimize the MSE (medium square error) cost function (35) for each tested dimension $N$ separately. The proposed model was compared to the integer order model expressed by (15)-(26). Results are given in Table 1 The cost function (35) as a function of $N$ for both discussed models is shown in Fig. 44 and optimal parameters for the integer order model are given in Table 2, whereas optimal parameters for the non-integer order model are given in Table 3 The error of the model as a function of time and the length of the rod is shown in Figs. 7 and 8 , the space-time distribution of the temperature calculated with the use of both the considered models is presented in Figs. 5 and 6 We define

$$
I_{\mathrm{MSE}}=\sum_{j=1}^{4} \int_{0}^{T_{f}}\left(y_{j}(t)-y_{e j}(t)\right)^{2} \mathrm{~d} t,
$$

where $y_{e j}(t)$ stands for data from the real plant.

From Table 1 and Fig. 4 we can conclude that the proposed non-integer order model is more accurate in the sense of the MSE cost function (35) for each discussed dimension of approximant $N$. Next, good accuracy is reached for the approximation order $N=15$, which gives

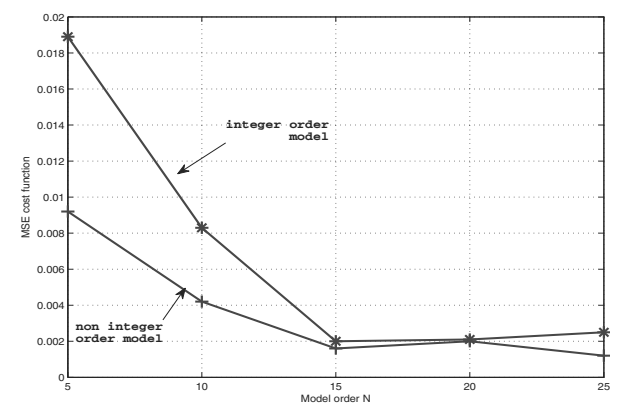

Fig. 4. Cost function (35) as a function of $N$.

Table 1. Cost function (35) for both models considered and different $N$.

\begin{tabular}{|c|c|c|}
\hline$N$ & integer order model & non-integer order model \\
\hline \hline 5 & 0.0189 & 0.0092 \\
\hline 10 & 0.0083 & 0.0042 \\
\hline 15 & 0.0020 & 0.0016 \\
\hline 20 & 0.0021 & 0.0020 \\
\hline 25 & 0.0025 & 0.0012 \\
\hline
\end{tabular}

Table 2. Optimal parameters of the integer order model for different $N$

\begin{tabular}{|c|c|c|}
\hline$N$ & $a_{w}$ & $R_{a}$ \\
\hline \hline 5 & 0.0017 & 0.0224 \\
\hline 10 & 0.0012 & 0.0253 \\
\hline 15 & 0.0009 & 0.0317 \\
\hline 20 & 0.0009 & 0.0312 \\
\hline 25 & 0.0009 & 0.0308 \\
\hline
\end{tabular}

Table 3. Optimal parameters of the non-integer order model for different $N$.

\begin{tabular}{|c|c|c|c|c|}
\hline$N$ & $\alpha$ & $\beta$ & $a_{w}$ & $R_{a}$ \\
\hline \hline 5 & 1.0571 & 2.1643 & 0.0012 & 0.0173 \\
\hline 10 & 1.0328 & 2.0526 & 0.0010 & 0.0224 \\
\hline 15 & 0.9896 & 1.9930 & 0.0009 & 0.0343 \\
\hline 20 & 0.9982 & 2.0018 & 0.0009 & 0.0316 \\
\hline 25 & 0.9744 & 1.9864 & 0.0007 & 0.0423 \\
\hline
\end{tabular}

a resonable model size during its application.

\section{Final conclusions}

Finally, we can conclude that the proposed non-integer order, state space model for the one dimensional heat plant can be built via generalization of an integer order, abstract model in the Hilbert space with the use of Feller semigroups. Furthermore, the accuracy of the proposed model in the sense of the MSE cost function is better than that of the analogical integer order model for each dimension of its finite dimensional approximation. The parameters of the proposed model can be estimated with the use of experimental results. 


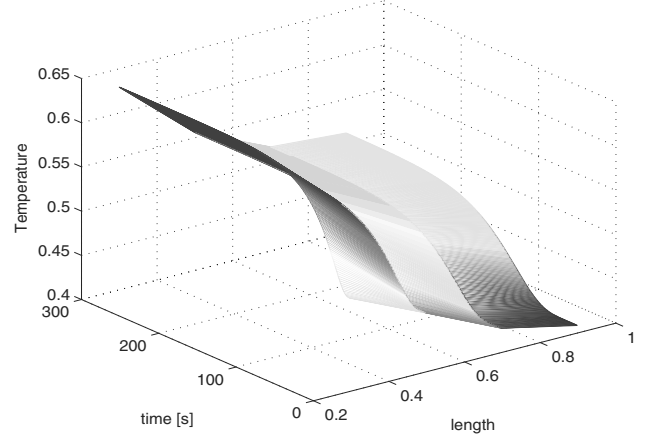

Fig. 5. Spatiotemporal temperature distribution for the integer order model and $N=15$.

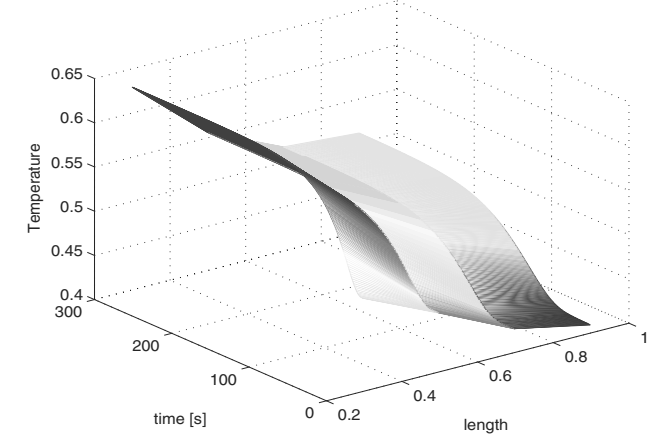

Fig. 6. Spatiotemporal temperature distribution for the noninteger order model and $N=15$.

Further investigations of the presented problems will cover generalization of the results to cover the uncertainty in parameters as well as formulating and solving spectrum decomposition conditions for the given class of infinite-dimensional, non-integer order systems.

\section{Acknowledgment}

This work was partially financed within the AGH UST projects no. 11.11.120.815 and 11.11.120.817.

\section{References}

Almeida, R. and Torres, D.F.M. (2011). Necessary and sufficient conditions for the fractional calculus of variations with Caputo derivatives, Communications in Nonlinear Science and Numerical Simulation 16(3): 1490-1500.

Baeumer, B., Kurita, S. and Meerschaert, M. (2005). Inhomogeneous fractional diffusion equations, Fractional Calculus and Applied Analysis 8(4): 371-386.

Balachandran, K. and Divya, S. (2014). Controllability of nonlinear implicit fractional integrodifferential systems, International Journal of Applied Mathematics and Computer Science 24(4): 713-722, DOI: 10.2478/amcs-2014-0052.

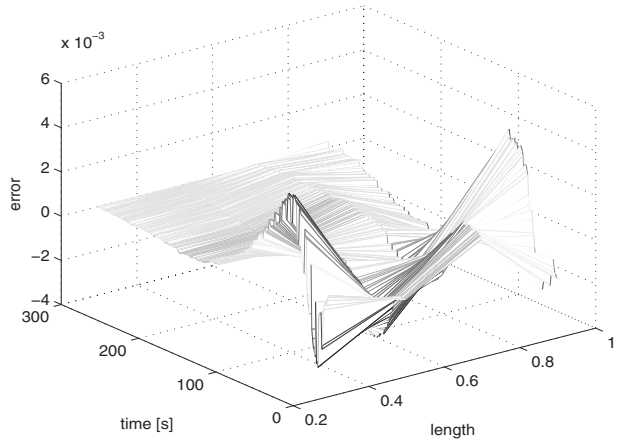

Fig. 7. Integer order model error as a function of time and the length of the rod for $N=15$.



Fig. 8. Non-integer order model error as a function of time and the length of the rod for $N=15$.

Balachandran, K. and Kokila, J. (2012). On the controllability of fractional dynamical systems, International Journal of Applied Mathematics and Computer Science 22(3): 523-531, DOI: 10.2478/v10006-012-0039-0.

Bartecki, K. (2013). A general transfer function representation for a class of hyperbolic distributed parameter systems, International Journal of Applied Mathematics and Computer Science 23(2): 291-307, DOI: 10.2478/amcs-2013-0022.

Caponetto, R., Dongola, G., Fortuna, L. and Petras, I. (2010). Fractional, order systems: Modeling and control applications, in L.O. Chua (Ed.), World Scientific Series on Nonlinear Science, University of California, Berkeley, CA, pp. 1-178.

Curtain, R.F. and Zwart, H. (1995). An Introduction to InfiniteDimensional Linear Systems Theory, Springer-Verlag, New York, NY.

Das, S. (2010). Functional Fractional Calculus for System Identification and Control, Springer, Berlin.

Dlugosz, M. and Skruch, P. (2015). The application of fractional-order models for thermal process modelling inside buildings, Journal of Building Physics 1(1): 1-13.

Dzielinski, A., Sierociuk, D. and Sarwas, G. (2010). Some applications of fractional order calculus, Bulletin of the Polish Academy of Sciences: Technical Sciences 58(4): 583-592. 
Evans, K.P. and Jacob, N. (2007). Feller semigroups obtained by variable order subordination, Revista Matematica Complutense 20(2): 293-307.

Gal, C. and Warma, M. (2016). Elliptic and parabolic equations with fractional diffusion and dynamic boundary conditions, Evolution Equations and Control Theory 5(1): 61-103.

Kaczorek, T. (2011). Selected Problems of Fractional Systems Theory, Springer, Berlin.

Kaczorek, T. (2016). Reduced-order fractional descriptor observers for a class of fractional descriptor continuous-time nonlinear systems, International Journal of Applied Mathematics and Computer Science 26(2): 277-283, DOI: 10.1515/amcs-2016-0019.

Kaczorek, T. and Rogowski, K. (2014). Fractional Linear Systems and Electrical Circuits, Białystok University of Technology, Białystok.

Kochubei, A. (2011). Fractional-parabolic systems, arXiv: 1009.4996 [math.ap], (reprint).

Mitkowski, W. (1991). Stabilization of Dynamic Systems, WNT, Warsaw, (in Polish).

Mitkowski, W. (2011). Approximation of fractional diffusion-wave equation, Acta Mechanica et Automatica 5(2): 65-68.

N'Doye, I., Darouach, M., Voos, H. and Zasadzinski, M. (2013). Design of unknown input fractional-order observers for fractional-order systems, International Journal of Applied Mathematics and Computer Science 23(3): 491-500, DOI: 10.2478/amcs-2013-0037.

Obraczka, A. (2014). Control of Heat Processes with the Use of Non-integer Models, Ph.D. thesis, AGH University of Science and Technology, Kraków.

Oprzedkiewicz, K. (2003). The interval parabolic system, Archives of Control Sciences 13(4): 415-430.

Oprzedkiewicz, K. (2004). A controllability problem for a class of uncertain parameters linear dynamic systems, Archives of Control Sciences 14(1): 85-100.

Oprzędkiewicz, K. (2005). An observability problem for a class of uncertain-parameter linear dynamic systems, International Journal of Applied Mathematics and Computer Science 15(3): 331-338.

Ostalczyk, P. (2012). Equivalent descriptions of a discrete-time fractional-order linear system and its stability domains, International Journal of Applied Mathematics and Computer Science 22(3): 533-538, DOI: 10.2478/v10006-012-0040-7.

Pazy, A. (1983). Semigroups of Linear Operators and Applications to Partial Differential Equations, Springer, New York, NY.

Podlubny, I. (1999). Fractional Differential Equations, Academic Press, San Diego, CA.

Popescu, E. (2010). On the fractional Cauchy problem associated with a Feller Semigroup, Mathematical Reports 12(2): 181-188.
Sierociuk, D., Skovranek, T., Macias, M., Podlubny, I., Petras, I., Dzielinski, A. and Ziubinski, P. (2015). Diffusion process modeling by using fractional-order models, Applied Mathematics and Computation 257(1): 2-11.

Szekeres, B.J. and Izsak, F. (2014). Numerical solution of fractional order diffusion problems with Neumann boundary conditions, preprint, arXiv: 1411.1596, [math.NA], (preprint).

Yang, Q., Liu, F. and Turner, I. (2010). Numerical methods for fractional partial differential equations with Riesz space fractional derivatives, Applied Mathematical Modelling 34(1): 200-218.

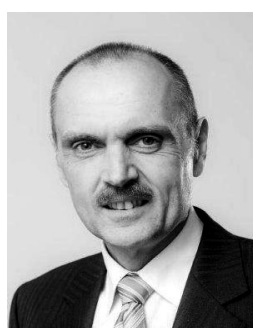

Krzysztof Oprzedkiewicz was born in Kraków (Poland) in 1964. He obtained an M.Sc. in electronics in 1988, and a Ph.D. and a D.Sc. in automatics and robotics in 1995 and 2009, respectively, at the AGH University of Science and Technology (Kraków). He has been working at the AGH University in the Department of Automatics since 1988 , recently as a professor and the vice dean of the Faculty of Electrotechnics, Automatics, Informatics and Biomedical Engineering. His research covers infinite dimensional systems, fractional order modeling and control, uncertain parameter systems, industrial automation, PLC and SCADA systems.

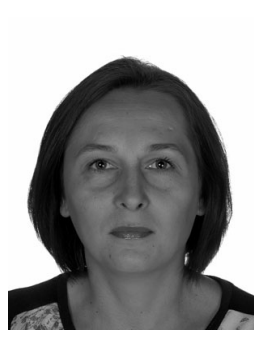

Edyta Gawin received her M.Sc. in mathematics at Jagiellonian University and a B.Sc. in informatics at the High Vocational School in Tarnów, Poland, in 2005 and 2003, respectively. She is currently preparing for a Ph.D. at the AGH University of Science and Technology, Faculty of Electrical Engineering, Automatics, Computer Science and Electronics. Her research interests cover technical applications of mathematics (e.g. in automatic control).

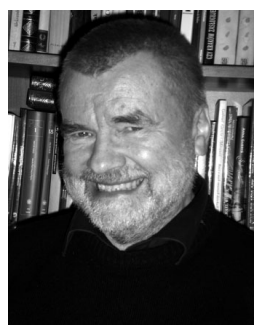

Wojciech Mitkowski was born in 1946 in Kraków. In 1970 he obtained an M.Sc. in electrical engineering in automation at the Faculty of Electrical Engineering at the AGH University of Science and Technology in Kraków, Poland. He has been working in this department ever since. He obtained a Ph.D. at the same faculty in 1974 and a D.Sc. in automatic control and robotics in 1984. The President of Poland awarded him the title of a professor of technical sciences in 1992. He has been a member of the Committee on Electrical Engineering, Computer Science and Control of the Polish Academy of Sciences, Kraków Branch, since 1988. He has also been a member of the Committee on Automatic Control and Robotics of the Polish Academy of Sciences since 1996. Between 2005 and 2010 he was the head of the Kraków Branch of the Polish Mathematical Society. His research interests include automation and robotics, control theory, optimal control, dynamic systems, circuits theory, numerical methods and applications of mathematics. He has published 9 books and 238 scientific papers.

Received: 15 December 2015 Revised: 13 April 2016 Re-revised: 11 July 2016 Accepted: 28 August 2016 